\title{
Review of: "A Sheathed Spike Gene, TaWUS-like Inhibits Stem Elongation in Common Wheat by Regulating Hormone Levels"
}

\author{
Xinwu Pei ${ }^{1}$ \\ 1 Chinese Academy of Agricultural Sciences
}

Potential competing interests: The author(s) declared that no potential competing interests exist.

In this manuscript, Liu and his colleagues found a novel gene, WUSCHEL-related homeobox like.

Overexpression transgenic lines were obtained, and the plant height, the flag leaf sheaths, the length of the uppermost and secondary internode and the parenchyma cells was systematically analysied. After minor revision this manuscripts can reach the publication level.

\section{Minor points:}

1. In Plant materials and growth conditions, Thirty OE homozygous lines and WT plants were selected randomly to investigate the agronomic traits including 1000-grain weight, plant height, tillering number and stem length of the uppermost and secondary internode. But these data are not seen in the manuscript.

2. Provide information on the gene copy number of the OE homozygous lines used in this study.

3. In Changes of endogenous GA and BR levels in flag leaf and internodes part. The comparison of GA and BR levels between OE E homozygous lines and WT plants only provides the proportion, do you have specific data?

4. In TaWUS-like affected the expression of GA, BR pathway and cell division related genes in grains part, 19 genes that related to GA synthesis and signal transduction were mentioned, but In Fig 4 A, only 15 genes? 